\title{
Preventing large sojourn times using SMART scheduling
}

\author{
Misja Nuyens, Adam Wierman ${ }^{\dagger}$ and Bert Zwart $\ddagger \S$
}

October 10, 2006

\begin{abstract}
Recently, the class of SMART scheduling policies has been introduced in order to formalize the common heuristic of "biasing toward small jobs." We study the tail of the sojourn-time (response-time) distribution under both SMART policies and the Foreground-Background policy (FB) in the GI/GI/1 queue. We prove that these policies behave very well under heavy-tailed service times. Specifically, we show that the sojourn-time tail under all SMART policies and FB is similar to that of the service-time tail, up to a constant, which makes the SMART class superior to First-Come-First-Served (FCFS). In contrast, for light-tailed service times, we prove that the sojourn-time tail under FB and SMART is larger than that under FCFS. However, we show that the sojourn-time tail for a job of size $y$ under FB and all SMART policies still outperforms FCFS as long as $y$ is not too large.
\end{abstract}

Subject classifications: Queues: Priority, Limit Theorems. Probability: Stochastic model applications

Area of review: Stochastic models

*Department of Mathematics, Vrije Universiteit Amsterdam, De Boelelaan 1081, 1081 HV Amsterdam, The Netherlands, mnuyens@few.vu.nl

${ }^{\dagger}$ Computer Science Department, Carnegie Mellon University, 5000 Forbes Avenue Pittsburgh, PA, USA, acw@cs.cmu.edu

${ }^{\ddagger}$ CWI, P.O. Box 94079, 1090 GB Amsterdam

$\S$ Department of Mathematics \& Computer Science, Eindhoven University of Technology, P.O. Box 513, 5600 MB Eindhoven, The Netherlands, zwart@win.tue.nl 


\section{Introduction}

Scheduling policies (disciplines) that bias toward small job sizes (service requirements) have recently received attention across a number of computer application areas. For instance, variants of ShortestRemaining-Processing-Time (SRPT), Foreground-Background (FB), and Preemptive-Shortest-JobFirst (PSJF) have been suggested for use in web servers (Cherkasova (1998), Harchol-Balter et al. (2003), Rawat and Kshemkalyani (2003)), routers (Rai et al. (2004), Yang and Veciana (2002)), and databases (McWherter et al. (2004)). As a result of the attention given to size-based policies by computer systems researchers, there has been a resurgence in analytical work studying these policies, see Aalto, Ayesta and Nyberg-Oksanen (2004), Borst et al. (2003), Mandjes and Nuyens (2005), Núñez Queija (2002), and Wierman and Harchol-Balter (2003), with SRPT and FB dominating the literature. However, SRPT and FB are idealized versions of the policies implemented by practitioners. In particular, the intricacies of computer systems force the use of complex hybrid policies in practice, though these more complex policies are still built around the heuristic of "giving priority to small jobs," see McWherter et al. (2004), Rai et al. (2004), and Rawat and Kshemkalyani (2003). Thus, there exists a gap between the results provided by theoretical research and the needs of practitioners.

An emerging style of research attempts to bridge this gap by formalizing general scheduling heuristics (such as giving priority to small/large jobs) and analyzing the impact of these heuristics instead of analyzing the behavior of idealized individual policies. The analysis of these heuristic classifications provides both practical and theoretical benefits. Theoretically, such results add structure to the space of scheduling policies that cannot be obtained by analyzing individual policies. Practically, such results provide analyses for the hybrid policies that are implemented in practice.

One such heuristic classification is the SMART class, introduced in Wierman, Harchol-Balter and Osogami (2005), which formalizes the heuristic of "prioritizing small jobs." The SMART classification includes policies such as SRPT and PSJF, but does not include FB, Processor-Sharing (PS), or FirstCome-First-Served (FCFS). Much more detail on the SMART class is provided in Section 2. To this point, it has been proven that all SMART policies have mean sojourn time (response time) within a factor of two of optimal (Wierman, Harchol-Balter and Osogami (2005)). However, beyond the mean sojourn time, little is known about the behavior of SMART policies. Filling this gap is one goal of the current work.

Though the SMART classification does not include FB, there are many similarities between FB and SMART policies. At every point in time, FB shares the server evenly among all the jobs in the system with the smallest age (least attained service), so that small jobs will have the server mostly to themselves. Furthermore, under distributions with a so-called decreasing failure rate, the age of a job is a good indicator of its remaining size. Since FB attempts to bias toward small (remaining) 
job sizes without knowledge of job sizes, it can be viewed as a "poor man's SMART policy". However, without knowledge of job sizes, FB cannot bias as strongly towards small jobs as SMART policies. Therefore it is interesting to contrast the behavior of SMART policies with the behavior of FB .

In this work, we focus on the behavior of the sojourn-time tail of FB and SMART policies in a GI/GI/1 queue under both heavy-tailed and light-tailed service distributions. We characterize the likelihood of large sojourn times under FB and SMART policies. For these policies such an analysis is especially important because it can quell fears that large jobs suffer "starvation" as a result of the bias toward small jobs.

We prove two main results. First, we show that for a large class of heavy-tailed service distributions, both FB and SMART policies have a sojourn-time tail that is similar to that of the service distribution, up to a multiplicative constant (Theorem 3). Thus, FB and SMART policies have asymptotically optimal sojourn-time tails. This is encouraging since in many computer applications service distributions tend to be heavy-tailed, see Barford and Crovella (1998), Downey (2001), Leland et al. (1993), and Peterson (1996). Second, for a large class of light-tailed service distributions having no mass at the endpoint of the distribution, both FB and SMART policies have a sojourn-time tail that is equal (on a logarithmic scale) to that of the busy period, which can be far from optimal (Theorem 4). Interestingly, when the service distribution is allowed to have mass at the endpoint, FB still has a sojourn-time tail that is equal to that of the busy period, while some SMART policies can have a lighter tail. Theorems 3 and 4 illustrate a trade-off that seems to be a general tendency: policies that have (near) optimal sojourn-time tail behavior under heavy-tailed service distributions, can behave poorly under light-tailed service distributions. In particular, it seems unlikely that any policy can obtain the "best of both worlds."

A more detailed look at the picture sketched above reveals the following: the poor behavior of the sojourn time under SMART disciplines under light-tailed service distributions is merely caused by the behavior of the largest jobs. In fact, the tail behavior of the sojourn time of a job of size $y$ is still better than that under FCFS, provided that $y$ is not too large (Theorem 5). Using a policy from the SMART class is therefore especially attractive when the tail of the service-time distribution is not known in advance.

The results we have described so far illustrate the similarities of FB and SMART with respect to the sojourn-time tail under both heavy and light-tailed service distributions. However, one expects SMART policies to provide smaller sojourn times than FB, since FB does not use knowledge of the job sizes to make scheduling decisions. This expectation is confirmed in Theorem 6 where we show that in an M/GI/1 queue, the conditional sojourn time of a job of size $x$ is stochastically larger under FB than under any SMART policy.

Theorem 3 (for heavy-tailed service times) extends and unifies earlier results for M/G/1 FB and 
M/G/1 SRPT, which were proven by analytic methods, requiring explicit conditions for conditional moments of the sojourn time. Our proof is completely different: it consists of several probabilistic arguments utilizing ideas from large deviations theory for heavy tails. Consequently, we do not require the assumption on Poisson arrivals. Theorems 4 and 5 have been proven before for SRPT and M/G/1 FB. The proof of Theorems 4 and 5 is an extension of the method employed in Nuyens \& Zwart (2005).

This paper is organized as follows. We begin by introducing and further motivating the SMART classification in Section 2. Next, in Section 3, we introduce our notation and define the classes of service-time distributions we study. In Section 4 we present and discuss the main results of the paper. The analysis begins in Section 5 where we study the case of light-tailed service distributions, and continues in Section 6 where we study the case of heavy-tailed service distributions. Then, in Section 7, we restrict the analysis to the M/GI/1 setting and derive stochastic bounds relating the sojourn time under FB and SMART disciplines. Finally, we conclude in Section 8.

\section{The SMART class}

It is well known that policies that "give priority to small jobs" perform well with respect to the mean sojourn time. As we have already discussed, this idea has been fundamental to many computer systems applications ranging from web servers and routers to supercomputing centers and operating systems. However, although the same heuristic guides all these implementations, the policies that result differ due to (i) implementation restrictions and (ii) concerns about metrics other than mean sojourn time (e.g., avoiding starvation of large jobs). In particular, hybrid policies are used instead of the idealized policies prioritizing small jobs that are studied in the theoretical literature, such as SRPT and PSJF.

The SMART class formalizes the heuristic of "giving priority to small jobs" in order to provide "SMAll Response Times" using three simple properties described below. This class includes a range of hybrid policies other than those typically studied by theoreticians. To this point, all that has been proven about SMART policies is that they all have mean sojourn time within a factor of two of optimal in the M/GI/1 setting. In this paper, we further characterize SMART policies by obtaining the asymptotic behavior of the sojourn-time tails in a general setting. Together, these two results provide a strong characterization of policies that "prioritize small jobs." 


\begin{tabular}{|c|l|}
\hline \hline FB & $\begin{array}{l}\text { Foreground-Background preemptively serves those jobs that have received } \\
\text { the least amount of service so far. }\end{array}$ \\
\hline FCFS & First Come First Served serves jobs in the order they arrive. \\
\hline LCFS & $\begin{array}{l}\text { Last Come First Served non-preemptively serves the job that arrived the } \\
\text { most recently. }\end{array}$ \\
\hline LRPT & $\begin{array}{l}\text { Longest Remaining Processing Time preemptively serves the job in the sys- } \\
\text { tem with the largest remaining processing time. }\end{array}$ \\
\hline LJF & $\begin{array}{l}\text { Longest Job First non-preemptively serves the job in the system with the } \\
\text { largest original size. }\end{array}$ \\
\hline PLCFS & $\begin{array}{l}\text { Preemptive Last Come First Served preemptively serves the most recent } \\
\text { arrival. }\end{array}$ \\
\hline PLJF & $\begin{array}{l}\text { Preemptive Longest Job First preemptively serves the job in the system with } \\
\text { the largest original size. }\end{array}$ \\
\hline PS & \begin{tabular}{l} 
Processor Sharing serves all customers simultaneously, at the same rate. \\
\hline PSJF
\end{tabular} $\begin{array}{l}\text { Preemptive Shortest Job First preemptively serves the job in the system } \\
\text { with the smallest original size. }\end{array}$ \\
\hline RS & $\begin{array}{l}\text { RS preemptively serves the job with the smallest product of remaining size } \\
\text { and original size. }\end{array}$ \\
\hline SJF & $\begin{array}{l}\text { Shortest Job First non-preemptively serves the job in the system with the } \\
\text { smallest original size. }\end{array}$ \\
\hline SRPT & $\begin{array}{l}\text { Shortest Remaining Processing Time preemptively serves the job with the } \\
\text { shortest remaining service requirement. }\end{array}$ \\
\hline \hline
\end{tabular}

Table 1: A brief description of the scheduling policies discussed in this paper.

\subsection{Defining SMART scheduling}

In Wierman, Harchol-Balter and Osogami (2005), the class of SMART policies is defined as follows. In the definition, we denote jobs by $a, b$, or $c$, where job $a$ has remaining size $r_{a}$ and original size $s_{a}$. We also define job $a$ to have priority over job $b$ if job $b$ can never run while job $a$ is in the system.

Definition 1 A work conserving policy $\mathrm{P}$ belongs to the class SMART, denoted $\mathrm{P} \in \mathrm{SMART}$, if it obeys the following properties.

Bias Property: If $r_{b}>s_{a}$, then job a has priority over job $b$. 


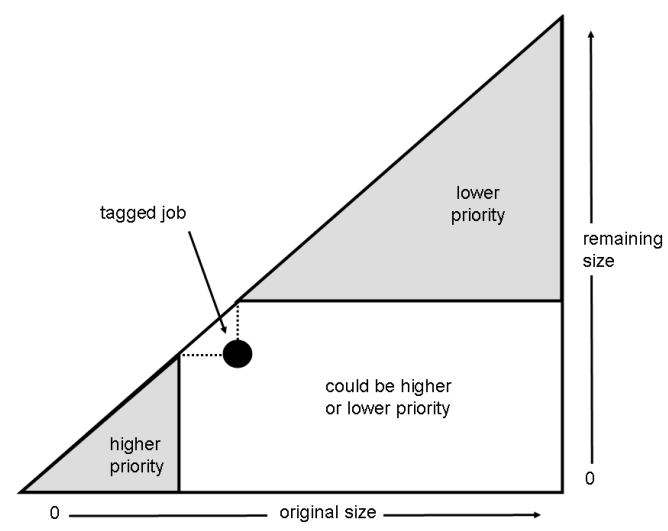

Figure 1: This diagram illustrates the priority structure induced by the Bias Property in Definition 1. The Consistency and Transitivity properties guarantee that, upon arrival, a job will find at most one job with higher priority in the white region (see Wierman, Harchol-Balter and Osogami (2005)). This property is important in many of our proofs.

Consistency Property: If job a ever receives service while job b is in the system, then at all times thereafter job a has priority over job b.

Transitivity Property: If an arriving job $b$ preempts job $c$, then thereafter, until job $c$ receives service, every arrival a with size $s_{a}<s_{b}$ is given priority over job $c$.

This definition has been crafted to mimic the heuristic of biasing towards jobs that are (originally) short or have small remaining service requirements. Each of the three properties formalizes a notion of "smart" scheduling. The Bias Property guarantees that the job being run at the server has remaining size smaller than the original size of all jobs in the system. In particular, this implies that the server will never work on a new arrival of size greater than $x$ while a previous arrival of original size $x$ is in the system. The priority structure enforced by the Bias Property is illustrated in Figure 1.

The Consistency and Transitivity Properties ensure coherency in the priority structure enforced by the Bias Property. In particular, the Consistency Property prevents time-sharing by guaranteeing that after job $a$ is chosen to run ahead of $b$, job $b$ will never run ahead of job $a$. Said a different way, this means that once job $a$ is given priority over job $b$, job $a$ will forever have priority over $b$. This makes intuitive sense because our priority function is based on the heuristic of giving priority to small jobs, and as job a receives service, it can only get smaller. Finally, the Transitivity Property guarantees that SMART policies do not second guess themselves: if an arrival $a$ is estimated to be "smaller" than job $b$ (and hence is given priority over job $b$ ), future arrivals smaller than $a$ 
are also considered "smaller" than $b$ until $b$ receives service.

\subsection{Examples of SMART scheduling}

Figure 2 provides a diagram illustrating where many of the commonly studied scheduling disciplines fall in relation to SMART. Brief descriptions of these policies are provided in Table 1.

Of course, the SMART class includes SRPT and PSJF. Further, it is easy to see that the SMART class includes the RS policy, which assigns to each job the product of its remaining size and its original size and then gives highest priority to the job with the smallest product. RS is an interesting policy because in many cases it outperforms SRPT with respect to weighted sojourn-time measures such as the slowdown, i.e., the sojourn time of a job divided by the size of the job. In addition, the SMART class includes many generalizations of these policies. Specifically, Wierman, Harchol-Balter, and Osogami (2005) show that $\mathrm{P} \in \mathrm{SMART}$ if $\mathrm{P}$ schedules the job with the lowest priority and gives each job of size $s$ and remaining size $r$ a priority using a fixed priority function $p(s, r)$ such that for $s_{1} \leq s_{2}$ and $r_{1}<r_{2}, p\left(s_{1}, r_{1}\right)<p\left(s_{2}, r_{2}\right)$. Thus, examples of SMART policies include $p(s, r)=s^{i} r^{j}$ for all $i \geq 0$ and $j>0$.

Given the range of performance metrics used in modern computer systems, it is of practical importance that SMART includes such a wide range of static priority policies. In particular, systems typically need to perform well for a combination of metrics, e.g., mean sojourn time, mean slowdown, and sojourn-time tail. For many of these metrics, the optimal SMART policy is not SRPT or PSJF, but it depends on the service distribution. For instance, no single SMART policy can optimize the mean slowdown across all service distributions, thus the best choice for optimizing a combination of mean sojourn time and mean slowdown depends on the service distribution. A key motivation for characterizing the class as a whole instead of studying the individual policies in the class is that no single SMART policy is optimal for all applications.

Apart from static priority policies, SMART also includes time-varying policies, i.e., policies than can change their priority rules over time, based on system-state information, or randomization. These generalizations are possible because the SMART definition enforces only a partial ordering on priorities of jobs in the system, see Wierman, Harchol-Balter, and Osogami (2005) for more detail. It is of enormous practical importance that time-varying policies are included in SMART, because it allows system designers to use the SMART class in order to perform online multi-objective optimization. Specifically, suppose a system designer wants to optimize a secondary objective while still providing small mean sojourn times. In order to accomplish this, the system designer can implement a parameterized version of SMART, such as prioritizing based on $p(s, r)=s^{i} r^{j}$, and then use machine learning techniques to search the space $(i, j)$ online for the SMART policy that optimizes the secondary objective. (Note that $i$ and $j$ can be chosen to achieve SRPT, PSJF, RS, 


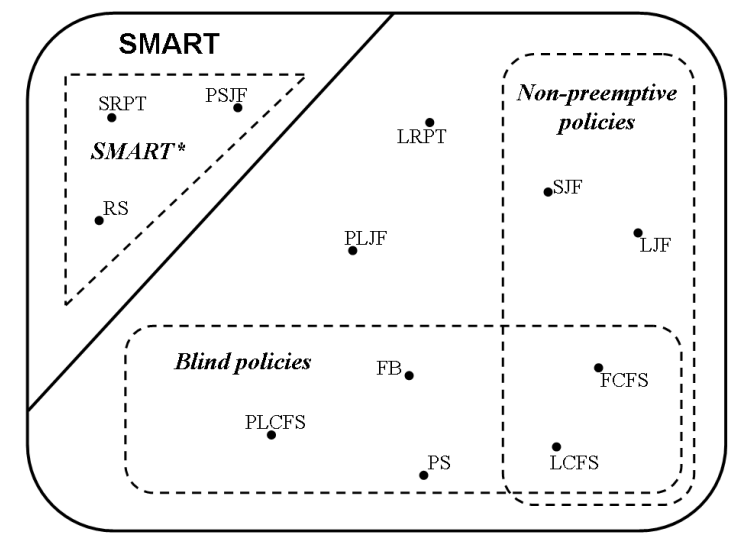

Figure 2: An illustration of the relation of many common scheduling disciplines to the SMART class.

and many other policies.) This technique can be extremely useful in web applications where the service distribution is time-varying and thus the optimal scheduling policy is not static. Because SMART includes time-varying policies, the bounds on the mean sojourn time from prior work and on the tail of sojourn time proven here will hold even as the priority function varies. The inclusion of online optimization policies is another key benefit of studying the SMART class as a whole, as analysis of such policies is absent from the literature. The inclusion of these policies complicates many of the analysis beyond what is necessary for static priority policies such as SRPT and PSJF.

\subsection{Policies excluded from SMART}

To this point we have only discussed the breadth of SMART. However, it is also important to note that many policies are excluded from SMART. Clearly, SMART does not include policies that give priority to large jobs such as LJF, PLJF, and LRPT. In addition, SMART does not include policies that only "weakly" prioritize small jobs. For example, SMART does not include any non-preemptive policies, not even ones like SJF that prioritize small jobs; nor does it include policies that do not use knowledge about the job sizes (blind policies), not even FB.

The exclusion of these policies is a result of the tension between the breadth of the class and the tightness of the results provable about the class. In particular, excluding policies such as SJF and FB that bias weakly towards small job sizes is necessary for SMART policies to have a near optimal mean sojourn time across all service distributions and all loads. For example, though SJF can provide good mean sojourn time when the second moment of the service distribution, $E\left[B^{2}\right]$, is small, the mean response time of SJF is arbitrarily larger than the optimal as $E\left[B^{2}\right] \rightarrow \infty$. Similarly, though FB can provide near optimal $E[V]$ under service distributions having decreasing failure rates, when 
the service distribution has an increasing failure rate, FB is one of the worst disciplines to use, see Righter and Shanthikumar (1989). In particular, when the service distribution is deterministic, the quotient $E[V]^{\mathrm{FB}} / E[V]^{\mathrm{SRPT}}$ can be arbitrarily large, see Nuyens (2004).

The tension between the breadth and tightness of the class also leads to the exclusion of policies having only a finite number of priority levels: such policies violate the Bias Property. It is particularly unfortunate to exclude these policies because in many cases system designers simplify implementations by using only 5-10 priority levels without sacrificing too much performance in practice, see, e.g., Harchol-Balter et al. (2003). Generalizing SMART in a way that includes such policies while still guaranteeing tight bounds on the mean sojourn time is a difficult task that is a current topic of research.

\section{Preliminaries}

Throughout the paper, unless otherwise stated, we consider a stationary preemptive-resume GI/GI/1 queue with generic service time $B$, having $E[B]<\infty$, and generic interarrival time $A$. The system load $\rho$ satisfies $\rho=E[B] / E[A]<1$, and we assume that $P(A<B)>0$ (otherwise there is no queueing). Let $F$ be the service distribution and $\bar{F}=1-F$ its tail. Define its (right) endpoint $x_{F} \stackrel{\text { def }}{=} \sup \{x: F(x)<1\}$. Define $f(x) \sim g(x)$ to mean that $\lim _{x \rightarrow \infty} f(x) / g(x)=1$. Let $\Phi_{X}$ denote the moment generating function of a random variable $X$, i.e., $\Phi_{X}(s)=E\left[e^{s X}\right]$.

Let $V^{\mathrm{P}}$ denote a random variable that is distributed according to the sojourn time in a stationary system under policy P. The sojourn time (response time) is the time between the arrival and the departure of a job. Let $V(x)^{\mathrm{P}}$ be distributed according to the sojourn time of a job of size $x$ in a stationary system under policy $\mathrm{P}$. The random variable $W^{\mathrm{P}}$, called the waiting time, is distributed as the time a job waits before its service starts in the stationary system; $W(x)^{\mathrm{P}}$ denotes the waiting time of a job of size $x$. Finally, let $R^{\mathrm{P}}$, the residence time of a job, be distributed as the time a job spends in the stationary system after his service has started, and let $R(x)^{\mathrm{P}}$ denote the residence time of a job of size $x$. Hence, we may write

$$
V(x)^{\mathrm{P}} \stackrel{d}{=} R(x)^{\mathrm{P}}+W(x)^{\mathrm{P}} .
$$

We consider two classes of service distributions in this work. The class of heavy-tailed distributions that we study are those of intermediate regular variation at infinity:

Definition 2 We say that the tail $\bar{F}(x)$ of a distribution is of intermediate regular variation at infinity, $\bar{F} \in \mathcal{I} \mathcal{R}$, when

$$
\liminf _{\varepsilon \downarrow 0} \liminf _{x \rightarrow \infty} \frac{\bar{F}(x(1+\varepsilon))}{\bar{F}(x)}=1
$$


This class includes all regularly varying tails and thus includes, for example, Pareto distributions.

The light-tailed distributions that we study obey one or both of the following assumptions:

Assumption A: $\Phi_{B}(s)<\infty$ for some $s>0$.

Assumption B: $P\left(B=x_{F}\right)=0$.

Note that the distributions that satisfy both of these assumptions include light-tailed distributions with infinite endpoints (e.g., exponential, gamma, and certain Weibull distributions), as well as all continuous distributions with finite support (e.g., uniform and beta distributions).

When studying the case of light-tailed service, we will describe the logarithmic behavior of the tail of the sojourn time distribution using the decay rate.

Definition 3 The (asymptotic) decay rate $\gamma(X)$ of a random variable $X$ is defined by

$$
\gamma(X)=\lim _{x \rightarrow \infty} \frac{-\log P(X>x)}{x}
$$

given that the limit exists.

Informally, for large $x$, one may write $P(X>x) \approx e^{-\gamma(X) x}$. It should be noted that a smaller decay rate corresponds to a larger tail of the distribution.

In both the light and heavy-tailed case, our analysis will depend heavily on the use of the following types of busy periods. Denote by $L$ a random variable with the steady-state busy-period distribution. Let $L(y)$ be a random variable with the same distribution as a steady-state busy period that is started by a job of size $y$. Let $L^{*}$ be distributed as the length of the steady-state busy period starting with the amount of work $Q+B$, i.e., $L^{*} \stackrel{d}{=} L(Q+B)$, where $Q$ is the steadystate amount of work in the system (upon customer arrivals), and $L(\cdot), Q$ and $B$ are independent. We call $L^{*}$ the residual busy period.

Let $L_{x}$ be distributed as the length of a steady-state busy period in the queue with generic service time $B I(B<x)$. So, in this queue, the service time of a customer is zero with probability $P(B \geq x)$. We assume that those customers leave the queue immediately. Hence, the busy period $L_{x}$ is made up of arrivals with service times less than $x$. Furthermore, let $L_{x}(y)$ be distributed as a busy period in the queue with service time $B I(B<x)$ that is started by a job of size $y$. Finally, let $\widetilde{L_{x}}$ be the length of a busy period with job sizes $B \wedge x$, where $x \wedge y \stackrel{\text { def }}{=} \min (x, y)$, and define $\widetilde{L_{x}}(y)$ similarly.

The decay rate of the busy period can be expressed in terms of the moment generating functions of $A$ and $B$. The following result is taken from Nuyens and Zwart (2005).

Lemma 1 The decay rate of the busy period satisfies:

$$
\gamma(L)=\sup _{s \geq 0}\left[s+\Phi_{A}^{\overleftarrow{A}}\left(\frac{1}{\Phi_{B}(s)}\right)\right]
$$


where $\Phi_{A}^{\leftarrow}$ is the inverse of $\Phi_{A}$. In particular, for $0<x \leq \infty$

$$
\begin{aligned}
& \gamma\left(L_{x}\right)=\sup _{s \geq 0}\left[s+\Phi_{A}^{\leftarrow}\left(\frac{1}{\Phi_{B I(B<x)}(s)}\right)\right], \\
& \gamma\left(\widetilde{L_{x}}\right)=\sup _{s \geq 0}\left[s+\Phi_{A}^{\leftarrow}\left(\frac{1}{\Phi_{(B \wedge x)}(s)}\right)\right] .
\end{aligned}
$$

The expressions for $\gamma(L), \gamma\left(L_{x}\right)$ and $\gamma\left(\widetilde{L_{x}}\right)$ can in general be solved numerically. If the arrival process is Poisson with rate $\lambda$ say, we get $\gamma(L)=\sup _{s \geq 0}\left[s-\lambda\left(\Phi_{B}(s)-1\right)\right]$. Specializing this to the $M / M / 1$ queue, where the service times have an exponential distribution with rate $\mu$, we get the explicit expression $\gamma(L)=\mu(1-\sqrt{\rho})^{2}$.

Since $B>B \wedge x$ and $B \wedge x>B I(B<x)$ with positive probability for all $x<x_{F}$, it is intuitively obvious that $\gamma(L)<\gamma\left(\widetilde{L_{x}}\right)<\gamma\left(L_{x}\right)$. We close the section with a short proof of this result.

Proposition 2 If $x<x_{F}$, then $\gamma(L)<\gamma\left(\widetilde{L_{x}}\right)$. If in addition $x$ is such that $P(A<x)>0$, then $\gamma\left(\widetilde{L_{x}}\right)<\gamma\left(L_{x}\right)$.

Proof Since $P(A<B)>0$, it follows from the proof of Proposition 2.2 in Nuyens and Zwart (2005) that the supremum in (1) is attained for some $s^{*} \in(0, \infty)$. Now, note that $B \wedge x<B$ with positive probability. This implies that $\Phi_{B}(s)>\Phi_{(B \wedge x)}(s)$. Since $\Phi_{A}(s)$ is strictly increasing and continuous in $s$, so is its inverse $\Phi_{A}^{\leftarrow}(s)$. Therefore,

$$
\gamma(L)=s^{*}+\Phi_{A}^{\leftarrow}\left(\frac{1}{\Phi_{B}\left(s^{*}\right)}\right)<s^{*}+\Phi_{A}^{\leftarrow}\left(\frac{1}{\Phi_{(B \wedge x)}\left(s^{*}\right)}\right) \leq \gamma\left(\widetilde{L_{x}}\right)
$$

This proves the first statement. The proof of the second statement follows the same lines. The assumption $P(A<x)>0$ implies that $P(A<B \wedge x)>0$, which guarantees the existence of an optimizing argument in (2).

\section{Results and discussion}

The focus of this work is to understand the distributional behavior of the sojourn time under SMART policies and to contrast this behavior with that of sojourn times under FB. To this end, we prove three main results: (i) we characterize the sojourn-time tail under a class of heavy-tailed service distributions, (ii) we characterize the sojourn-time tail under a class of light-tailed distributions, and (iii) we prove that the sojourn-time distribution of FB is stochastically larger than that of any SMART policy. In the remainder of this section we will state and discuss these results.

Theorem 3 In the GI/GI/1 queue with $\mathrm{P} \in \mathrm{SMART}$ or $\mathrm{P}=\mathrm{FB}$, if $\bar{F} \in \mathcal{I} \mathcal{R}$, then

$$
P\left(V^{\mathrm{P}}>x\right) \sim P(B>(1-\rho) x), \text { as } x \rightarrow \infty .
$$


Theorem 3 characterizes the sojourn-time tail of SMART and FB under heavy-tailed service distributions. Since SRPT $\in$ SMART, Theorem 3 can be viewed as a generalization of the recent results that show that relation (3) holds in the M/GI/1 setting for SRPT (Núñez Queija (2002)) and for FB (Núñez Queija (2002) and Nuyens (2004)). Furthermore, relation (3) has been shown to hold for a number of queues with the processor sharing discipline, see Borst, Núñez Queija and Zwart (2006) for an overview.

Although it has not been proven, the sojourn-time tail of SMART policies seems to be optimal: there seem to be no policies with a smaller tail of the sojourn-time distribution than the one described in (3). In any case, this behavior is "near optimal" in the sense that no policy can have a sojourn time tail more than a multiplicative constant smaller. Furthermore, for heavy-tailed distributions, the tail of the sojourn time under a SMART policy is much smaller than the tail under FCFS: for FCFS and all other non-preemptive policies, the sojourn-time tail is 'one degree heavier' than the tail of the service distribution, i.e., it is of the order $x P(B>x)$, see Borst et al. (2003) for a survey.

For the second main result, recall that $L$ is distributed as the length of a busy period in the stationary queue.

Theorem 4 In the GI/GI/1 queue with $\mathrm{P} \in \mathrm{SMART}$, if Assumption A holds, then

$$
\gamma(L)=\gamma\left(V^{\mathrm{FB}}\right) \leq \gamma\left(V^{\mathrm{P}}\right) \leq \gamma\left(V^{\mathrm{SRPT}}\right) .
$$

Furthermore, if both Assumptions $A$ and B hold, then $\gamma\left(V^{\mathrm{P}}\right)=\gamma\left(V^{\mathrm{FB}}\right)=\gamma(L)$. That is,

$$
\log P\left(V^{\mathrm{P}}>x\right) \sim \log P\left(V^{\mathrm{FB}}>x\right) \sim \log P(L>x), \text { as } x \rightarrow \infty .
$$

Theorem 4 characterizes the sojourn-time tail of SMART and FB under light-tailed service distributions. For light-tailed service times, $\gamma(L)$ is the smallest possible decay rate of the sojourn time, see Lemma 7. Hence, we can conclude that, on a logarithmic scale, the tail of $V$ under SMART disciplines can be as large as possible. Theorem 4 generalizes the result obtained in Mandjes and Nuyens (2005) for the M/GI/1/FB queue. Since SRPT $\in$ SMART, Theorem 4 can also be viewed as a generalization of a result in Nuyens and Zwart (2005), where it is shown that (4) holds under Assumptions A and B for GI/GI/1/SRPT. In addition, Nuyens and Zwart (2005) show that for distributions that satisfy Assumption A but not Assumption $\mathrm{B}$, the decay rate of $V^{\mathrm{SRPT}}$ in the GI/GI/1 queue lies strictly between that of $L$ and that of the stationary workload in the queue, which is equal to the decay rate of the sojourn time under FCFS. Relation (4) has recently been obtained for PS in Mandjes and Zwart (2004): in the GI/GI/1/PS queue, (4) holds for PS instead of FB under Assumptions A, B, and an additional condition that rules out service distributions with very light tails. 
The above theorems emphasize the similarities in the sojourn-time distribution of SMART and FB. However, if Assumption A holds and Assumption B does not hold, then the decay rate depends in a delicate way on the specific policy considered. The treatment of jobs with the same service time becomes crucial. That is, when jobs with the same service time are ordered in a FCFS manner (as in SRPT), the tail behavior can differ from when the server is shared among jobs with the same service time (as in FB).

It is important to note the contrast in the behavior of the sojourn-time tail of FB and SMART policies under heavy-tailed and light-tailed service distributions. This seems to be a general tendency: policies that behave (near) optimally for heavy-tailed service times, can behave very poorly for light-tailed distributions. This is a recurring theme in the literature: for example, Righter and Shanthikumar $(1989,1992)$ show a similar result for the queue length, although there the division is between service distributions with an increasing failure rate and those with a decreasing failure rate.

However, a disclaimer should be added: the poor behavior in the light-tailed case is merely caused by the sojourn times of the largest jobs. For smaller jobs, using FB or a SMART policy may not be so bad, as illustrated by the following theorem.

Theorem 5 Consider a GI/GI/1 queue under Assumption A. For y such that $P(B=y)=0$, we have for all $\mathrm{P} \in \mathrm{SMART}$,

$$
\gamma\left(V(y)^{\mathrm{P}}\right)=\gamma\left(L_{y}\right)
$$

Furthermore, for all $y>0$,

$$
\gamma\left(V(y)^{\mathrm{FB}}\right)=\gamma\left(\widetilde{L_{y}}\right)
$$

This result unifies earlier results for the GI/GI/1 SRPT and the M/GI/1 FB in Mandjes and Nuyens (2005) and Nuyens and Zwart (2005), and is important for several reasons. First of all, if $y$ is not too large, then $\gamma\left(L_{y}\right)$ is larger than $\gamma\left(V^{\mathrm{FCFS}}\right)$ as is illustrated in Nuyens and Zwart (2005). In particular, the threshold value $y^{*}$ for which $\gamma\left(L_{y^{*}}\right)=\gamma\left(V^{\mathrm{FCFS}}\right)$ converges to infinity if the traffic is either light $(\rho \rightarrow 0)$ or heavy $(\rho \rightarrow 1)$. Thus, under very high or low loads, one can still say that the SMART class outperforms FCFS under light-tailed service times. This illustrates the robustness of policies in the SMART class, which is important when the shape of the service-time distribution is not known in advance.

Since $\gamma\left(L_{y}\right)<\gamma\left(\widetilde{L_{y}}\right)$ for all $y<x_{F}$ by Proposition 2, Theorem 5 illustrates that the tail of the conditional response time is heavier under FB than under SMART policies. This is not surprising since FB does not use information about the job sizes or remaining sizes when scheduling. In fact, as we saw in Section 2, the difference between $E\left[V^{\mathrm{FB}}\right]$ and $E\left[V^{\mathrm{P}}\right]$ for $\mathrm{P} \in \mathrm{SMART}$ can be arbitrarily 
large. Another illustration that SMART policies outperform FB is given in Section 7, where we prove the following stochastic bound in the M/GI/1 setting:

Theorem 6 In an $M / G I / 1$ queue, for all $\mathrm{P} \in \mathrm{SMART}$ and all $x \geq 0$ :

$$
V(x)^{\mathrm{P}} \leq_{s t} R(x)^{\mathrm{PSJF}}+W(x)^{\mathrm{SRPT}} \leq_{s t} V(x)^{\mathrm{FB}} .
$$

\section{Light-tailed service demands}

In this section we prove Theorems 4 and 5 . Before starting with the proof of the theorems, we need two additional lemmas. First, we relate the decay rates of $L$ and $L^{*}$. This relation is trivial in the $M / G / 1$ queue, but for the $G I / G I / 1$ queue we need additional arguments.

Lemma 7 Under Assumption A, for all work-conserving disciplines $\mathrm{P}, \gamma\left(L^{*}\right)=\gamma(L)$. In particular, $\gamma\left(L_{x}^{*}\right)=\gamma\left(L_{x}\right)$ and $\gamma\left(\widetilde{L_{x}^{*}}\right)=\gamma\left(\widetilde{L_{x}}\right)$ for all $x \geq 0$.

Proof Let $V$ be the steady-state virtual waiting time in the $G I / G I / 1$ queue. Then $[L(V) \mid L(V)>$ $0] \stackrel{d}{=}[L(V) \mid V>0]$ has density $P(L>x) / E[L]$. Under Assumption A, Lemma 3.2 in Abate and Whitt (1997) yields that $L$ and $L(V)$ have the same decay rate. However, we are interested in the decay rate of $L(Q+B)$. In the $M / G / 1$ case, we could apply PASTA. In the general case, we note that $V \stackrel{d}{=}\left(Q+B-A^{*}\right)^{+}$, with $A^{*}$ a residual interarrival time. Therefore, $V$ is stochastically smaller than $Q+B$. Consequently, $\gamma\left(L^{*}\right)=\gamma(L(Q+B)) \leq \gamma(L(V))=\gamma(L)$. To prove the upper bound, let $A(t)$ be the total amount of work fed into the system between time 0 and $t$. Using the Chernov bound, we find for all $x \geq 0$ and $s \geq 0$ :

$$
P(L(Q+B)>x) \leq P(A(x)-x+Q+B>0) \leq E\left[e^{s Q}\right] E\left[e^{s B}\right] E\left[e^{s(A(x)-x)}\right] .
$$

Now (7) can be obtained by minimizing the last factor over $s$, and showing that for the optimizing argument $s^{*}$, we have $E\left[e^{s^{*} Q}\right]<\infty$ and $E\left[e^{s^{*} B}\right]<\infty$. Since this is exactly what is done in Proposition 3.1 of Mandjes and Zwart (2004), we refer to that work for the remaining supporting arguments. The proof is completed by noting that $L_{x}$ and $\widetilde{L_{x}}$ are GI/GI/1 busy periods themselves, for which we showed before that the residual busy period has the same decay rate.

The following lemma, which is Proposition 2.2 in Nuyens and Zwart (2005), will also play a key role in our arguments.

Lemma 8 For a GI/GI/1 queue under Assumption $A, \gamma\left(L_{x}\right) \downarrow \gamma(L)$ and $\gamma\left(\widetilde{L_{x}}\right) \downarrow \gamma(L)$ as $x \uparrow x_{F}$. 
Using these two preliminary lemmas we can begin to analyze the behavior of SMART. The techniques we apply are similar to those applied for SRPT in Nuyens and Zwart (2005).

We start by upper bounding the tail of $V^{\mathrm{P}}$ under all work-conserving disciplines $\mathrm{P}$ using the observation

$$
V^{\mathrm{P}} \leq_{s t} L^{*}
$$

Combining this observation with Lemma 7, we can lower bound $\gamma(V)$ by $\gamma(L)$ :

$$
\limsup _{x \rightarrow \infty} \frac{1}{x} \log P\left(V^{\mathrm{P}}>x\right) \leq-\gamma\left(L^{*}\right)=-\gamma(L) .
$$

We first do the analysis for the simplest case: both Assumptions A and B hold, and the service distribution is unbounded.

Lemma 9 In the GI/GI/1 queue with $\mathrm{P} \in \mathrm{SMART}$, if Assumptions $A$ and $B$ hold, and $x_{F}=\infty$, then $\gamma\left(V^{\mathrm{P}}\right)=\gamma(L)$. That is,

$$
\log P\left(V^{\mathrm{P}}>x\right) \sim \log P(L>x) \text {, as } x \rightarrow \infty .
$$

Proof Let $A_{1}$ be the first arrival after that of a tagged customer with size $B_{0}$. Let $a$ be such that $P\left(A_{1}<a\right)>0$ and $y<x_{F}-a$. Then for all $P \in$ SMART,

$$
\begin{aligned}
P\left(V^{\mathrm{P}} \geq x\right) & \geq P\left(V\left(B_{0}\right)^{\mathrm{P}}>x, A_{1}<a, B_{0}>y+a\right) \\
& =P\left(A_{1}<a, B_{0}>y+a\right) P\left(V\left(B_{0}\right)^{\mathrm{P}}>x \mid A_{1}<a, B_{0}>y+a\right) .
\end{aligned}
$$

Conditional on $B_{0}>y+a$ and $A_{1}<a$, the tagged job has remaining service time larger than $y$ when the new job arrives. The Bias Property implies that this new job has higher priority than the tagged job if its service time is smaller than $y$. Furthermore, all jobs with service time smaller than $y$ that arrive while the new job is in the system will also have higher priority than the tagged job. Thus, conditional on $B_{0}>y+a$ and $A_{1}<a$, we have $V\left(B_{0}\right)^{\mathrm{P}} \geq_{s t} L_{y}$. Hence,

$$
P\left(V^{\mathrm{P}} \geq x\right) \geq P\left(A_{1}<a, B_{0}>y+a\right) P\left(L_{y}>x\right) .
$$

Since $P\left(A_{1}<a, B_{0}>y+a\right)>0$, the existence of $\gamma\left(L_{y}\right)$ implies that

$$
\liminf _{x \rightarrow \infty} \frac{1}{x} \log P\left(V^{\mathrm{P}} \geq x\right) \geq \liminf _{x \rightarrow \infty} \frac{1}{x} P\left(L_{y}>x\right)=-\gamma\left(L_{y}\right) .
$$

To prove the lemma, it suffices to show that the liminf result corresponding to (8) holds. Letting $y$ go to $\infty$ in (9), and applying Lemma 8 , yields

$$
\liminf _{x \rightarrow \infty} \frac{1}{x} \log P\left(V^{\mathrm{P}} \geq x\right) \geq-\gamma(L) .
$$


This completes the proof.

Next, we relax the assumption that the service distribution is unbounded. This relaxation forces a more involved argument.

Lemma 10 In the GI/GI/1 queue with $\mathrm{P} \in \mathrm{SMART}$, if Assumptions $A$ and $B$ hold, and $x_{F}<\infty$, then $\gamma\left(V^{\mathrm{P}}\right)=\gamma(L)$.

Proof If $P(A<a)>0$ for all $a>0$, then the result follows from (9) and Lemma 8 , as in the proof of Lemma 9. However, this may not be the case, so we need a different construction.

By definition of $x_{F}$, there exists a decreasing sequence $\left\{\varepsilon_{n}\right\}$ such that $\varepsilon_{n} \rightarrow 0$ as $n \rightarrow \infty$, and $P\left(x_{F}-\varepsilon_{n}<B<x_{F}-\varepsilon_{n} / 2\right)>0$ for all $n$. The assumption $P(B>A)>0$ implies that $P\left(A<x_{F}\right)>0$. Hence, we may assume that $\varepsilon_{1}$ is such that $P\left(A<x_{F}-2 \varepsilon_{1}\right)>0$. Let $\lfloor x\rfloor$ denote the largest integer smaller than or equal to $x$. Let $Z_{n}$ be the event that the last $\left\lfloor x_{F} / \varepsilon_{n}\right\rfloor$ customers that arrived before the tagged customer had a service time in the interval $\left(x_{F}-\varepsilon_{n}, x_{F}-\varepsilon_{n} / 2\right)$, and that the last $\left\lfloor x_{F} / \varepsilon_{n}\right\rfloor$ inter-arrival times were smaller than $x_{F}-2 \varepsilon_{n}$. By definition of $\varepsilon_{n}$, we have $P\left(Z_{n}\right)>0$ for all $n$.

Furthermore, the Bias Property guarantees that, on the event $Z_{n}$, there is a customer with remaining service time larger than $k \varepsilon_{n}$ after the $k$ th of the inter-arrival times. Hence, at the arrival of the tagged customer (after $k=\left\lfloor x_{F} / \varepsilon_{n}\right\rfloor$ arrivals), there is a customer in the system with remaining service time in the interval $\left(x_{F}-\varepsilon_{n}, x_{F}-\varepsilon_{n} / 2\right)$. If the tagged customer has service time $B_{0}>x_{F}-\varepsilon_{n} / 2$, his sojourn time satisfies $V^{\mathrm{P}} \geq L_{x_{F}-\varepsilon_{n}}$. Consequently, for all $n \in \mathbb{N}$,

$$
P\left(V^{\mathrm{P}}>x\right) \geq P\left(Z_{n}\right) P\left(B_{0}>x_{F}-\varepsilon_{n} / 2\right) P\left(L_{x_{F}-\varepsilon_{n}}>x\right) .
$$

Thus, for $\mathrm{P} \in \mathrm{SMART}$, we have

$$
\liminf _{x \rightarrow \infty} \frac{1}{x} \log P\left(V^{\mathrm{P}}>x\right) \geq-\gamma\left(L_{x_{F}-\varepsilon_{n}}\right) .
$$

As $n \rightarrow \infty$, and hence $\varepsilon_{n} \downarrow 0$, Lemma 8 implies that $\gamma\left(L_{x_{F}-\varepsilon_{n}}\right) \rightarrow \gamma(L)$. Using (8) completes the proof.

We now turn to the analysis of the conditional sojourn time under SMART policies.

Lemma 11 In the GI/GI/1 queue, under Assumption $A$, if $P(B=y)=0$, then for all $\mathrm{P} \in \mathrm{SMART}$,

$$
\gamma\left(V(y)^{\mathrm{P}}\right)=\gamma\left(L_{y}\right) .
$$


Proof For the lower bound, we remark that $V(y)^{\mathrm{P}} \geq_{s t} L_{y}^{*}$ for all $\mathrm{P} \in$ SMART. By Lemma 7 , this residual busy period has decay rate $\gamma\left(L_{y}\right)$.

For the upper bound, we use Lemma 4.1 of Wierman, Harchol-Balter and Osogami (2005), which states that at any point in time, at most one customer with original service time larger than $y$ has remaining service time smaller than $y$. (Note that this lemma requires all three properties in the definition of SMART.) Denoting by $Q_{y}$ the stationary workload upon arrival instants, made up of customers with service time smaller than $y$, we can bound

$$
V(y)^{\mathrm{P}} \leq_{s t} L_{y}\left(Q_{y}+y+y\right) .
$$

Denoting the amount of work brought by customers (with size smaller than $y$ ) entering the queue in the interval $[0, x]$ by $A_{y}(x)$, the Chernov bound yields that for all $s \geq 0$,

$$
\begin{aligned}
P\left(V(y)^{\mathrm{P}}>x\right) & \leq P\left(L_{y}\left(Q_{y}+2 y\right)>x\right) \leq P\left(Q_{y}+2 y+A_{y}(x)>x\right) \\
& =P\left(\exp \left(s\left(Q_{y}+2 y+A_{y}(x)\right)>e^{s x}\right) \leq e^{-s x} e^{2 s y} E e^{s Q_{y}} E e^{s A_{y}(x)} .\right.
\end{aligned}
$$

Hence, for all $s<\gamma\left(Q_{y}\right)$, we have

$$
\limsup _{x \rightarrow \infty} \frac{1}{x} \log P\left(V(y)^{\mathrm{P}}>x\right) \leq-s+\limsup _{x \rightarrow \infty} \frac{1}{x} \log E e^{s A_{y}(x)}=-s-\Phi_{A}^{\leftarrow}\left(\frac{1}{\Phi_{B I(B<y)}(s)}\right),
$$

where the equality follows from Lemma 2.1 in Mandjes and Zwart (2004). Taking the infimum over all $s \in\left[0, \gamma\left(Q_{y}\right)\right)$ yields

$$
\limsup _{x \rightarrow \infty} \frac{1}{x} \log P\left(V(y)^{\mathrm{P}}>x\right) \leq-\sup _{0 \leq s<\gamma\left(Q_{y}\right)}\left[s+\Phi_{A}^{\leftarrow}\left(\frac{1}{\Phi_{B I(B<y)}(s)}\right)\right]=-\gamma\left(L_{y}^{*}\right),
$$

where the equality follows from equation (5.1) in Nuyens and Zwart (2005). By Lemma 7, $L_{y}$ and $L_{y}^{*}$ have the same decay rate. This yields the desired upper bound, and completes the proof.

The proof of Lemma 11 can be adapted to give the following result for FB .

Lemma 12 In the GI/GI/1 queue, under Assumption $A, \gamma\left(V(y)^{\mathrm{FB}}\right)=\gamma\left(\widetilde{L_{y}}\right)$ for all $y$. Furthermore, $\gamma\left(V^{\mathrm{FB}}\right)=\gamma(L)$.

Proof In the queue with generic service time $B \wedge y$, we have $V^{\mathrm{FB}} \stackrel{d}{=} \widetilde{L}_{y}^{*}$. Hence, $V^{\mathrm{FB}}(y) \geq_{s t}{\widetilde{L_{y}}}^{*}$. Furthermore, $V^{\mathrm{FB}}(y) \leq_{s t} L_{y}\left(Q_{y}+y\right)$, where $Q_{y}$ is the stationary workload in the queue upon arrival instants. Using these two bounds for $V^{\mathrm{FB}}(y)$, we can apply the proof of Lemma 11, replacing $B I(B<y)$ with $B \wedge y$, to get

$$
\gamma\left(V^{\mathrm{FB}}(y)\right)=\gamma\left(\widetilde{L}_{y}^{*}\right)
$$


The first statement now follows from Lemma 7. To prove the second part of the lemma, we consider two cases. If $P\left(B=x_{F}\right)>0$, then using (11) with $y=x_{F}$ and noting that $\widetilde{L_{x_{F}}}=L$ yields the desired result. If $P\left(B=x_{F}\right)=0$, then the result follows from applying Lemma 8 .

We are now ready to prove Theorems 4 and 5 .

Proof of Theorem 4 By Lemmas 9 and 10, we only need to deal with the case that Assumption B does not hold, i.e., $P\left(B=x_{F}\right)>0$. The first inequality follows from (8) and Lemma 12 . For the second inequality, note that $P\left(V^{\mathrm{P}}>x\right) \geq P\left(V\left(x_{F}\right)^{\mathrm{P}}>x\right) P\left(B=x_{F}\right)$. Thus, since $P\left(B=x_{F}\right)>0$, $\gamma\left(V^{\mathrm{P}}\right) \leq \gamma\left(V\left(x_{F}\right)^{\mathrm{P}}\right)$. Furthermore, for all $\mathrm{P} \in \mathrm{SMART}, V\left(x_{F}\right)^{\mathrm{P}} \geq_{s t} W\left(x_{F}\right)^{\mathrm{P}} \geq_{s t} W_{2}\left(x_{F}\right)$, where $W_{2}\left(x_{F}\right)$ is the waiting time of a low priority job in a 2-class preemptive priority queue where the high-priority class includes all jobs smaller than $x_{F}$. To complete the proof, we apply Theorems 3.1 and 4.2 of Nuyens and Zwart (2005), which state that $\gamma\left(W_{2}\left(x_{F}\right)\right)=\gamma\left(V^{\mathrm{SRPT}}\right)$.

Finally, the second statement of the theorem follows immediately from Lemmas 10 and 12

Note that, in contrast to FB, the sojourn-time tail of SMART policies can improve when there is mass in the endpoint of the service distribution. This is not surprising since many SMART policies, e.g., SRPT, are equivalent to FCFS in the GI/D/1 queue. However, SMART also includes policies where, like FB, jobs of the same size are not served in FCFS order. Thus, the SMART policies have a range of possible sojourn-time tails in this setting.

Proof of Theorem 5 The result follows directly from Lemmas 11 and 12.

\section{$6 \quad$ Heavy-tailed service demands}

In this section we prove Theorem 3, the result for heavy-tailed service times. Since Theorem 3 only assumes renewal arrivals, and not Poisson arrivals, it generalizes earlier results for M/G/1 FB (Mandjes and Nuyens (2005)) and M/G/1 SRPT (Nuyens and Zwart (2005)). The proofs of those results make use of explicit expressions for the conditional moments of the sojourn time. The proof of Theorem 3 is entirely different and consists of a sequence of probabilistic arguments.

The following theorem and sufficient conditions from Guillemin, Robert and Zwart (2004) form the core of the proof of Theorem 3, see also Borst, Núñez and Zwart (2006) where a.s. convergence in the next condition is weakened to convergence in probability:

Condition 1 For some $g>0, V(x)^{P} / x \rightarrow g$ in probability as $x \rightarrow \infty$. 
Condition 2 There exists a constant $k$ such that

$$
P\left(V(x)^{P}>k x\right)=o(\bar{F}(x))
$$

Theorem 13 If Conditions 1 and 2 hold, $\bar{F} \in \mathcal{I R}$, and $E\left[B^{p}\right]<\infty$ for some $p>1$, then

$$
P\left(V^{P}>g x\right) \sim P(B>x) \text { as } x \rightarrow \infty .
$$

We will prove Theorem 3 in two steps: first we show that Condition 1 holds for FB and all $\mathrm{P} \in \mathrm{SMART}$, and then that Condition 2 holds.

Lemma 14 For all $\mathrm{P} \in \mathrm{SMART}$ and $\mathrm{P}=\mathrm{FB}$, we have that $V(x)^{\mathrm{P}} / x \rightarrow 1 /(1-\rho)$ in probability as $x \rightarrow \infty$.

Proof We will prove the result by showing upper and lower bounds on the limit. All limits in this proof are taken as limits in probability.

To prove the lower bound, we first derive a stochastic lower bound for the sojourn time of a tagged customer in terms of a single busy period. For FB we have

$$
V(x)^{\mathrm{FB}} \geq_{s t} \widetilde{L_{x}}(x) \geq_{s t} L_{x}(x) \geq_{s t} L_{\varepsilon x}((1-\varepsilon) x), \quad 0<\varepsilon<1 .
$$

Furthermore, for $\mathrm{P} \in \mathrm{SMART}$, the Bias property guarantees that until the tagged job has received $(1-\varepsilon) x$ units of service, all arriving jobs smaller than $\varepsilon x$ receive priority. Hence,

$$
V(x)^{\mathrm{P}} \geq_{s t} L_{\varepsilon x}((1-\varepsilon) x), \quad 0<\varepsilon<1 .
$$

To understand the length of the busy period, we will analyze a PLCFS system. Define $S_{y}(t)$ to be the service given in the time interval $[0, t]$ to a permanent customer arriving in an empty queue at time 0 when the generic service time is $B I(B<y)$. Denoting the inverse of $L_{y}(\cdot)$ by $L_{y}^{\leftarrow}(\cdot)$, we have for all $x$ and $t$,

$$
P\left(S_{y}(t)>x\right)=P\left(L_{y}(x)<t\right)=P\left(L_{y}^{\leftarrow}(t)>x\right) .
$$

Hence, $S_{y}$ is stochastically equal to $L_{y}^{\leftarrow}$. This gives

$$
\lim _{x \rightarrow \infty} \frac{L_{y}(x)}{x}=\lim _{x \rightarrow \infty} \frac{x}{L_{y}^{\leftarrow}(x)} \stackrel{d}{=} \lim _{x \rightarrow \infty} \frac{x}{S_{y}(x)}=\frac{1}{1-\rho(y)}
$$

where $\rho(y)=E[B I(B<y)] / E[A]$. Now let $0<\varepsilon<1$, and $k>0$. Since $L_{y}$ is stochastically increasing in $y$, it follows from (12), (13) and (14) that for all $\mathrm{P}=\mathrm{FB}$ and $\mathrm{P} \in$ SMART,

$$
\liminf _{x \rightarrow \infty} \frac{V(x)^{\mathrm{P}}}{x} \geq_{s t} \lim _{x \rightarrow \infty} \frac{L_{\varepsilon x}((1-\varepsilon) x)}{x} \geq_{s t}(1-\varepsilon) \lim _{z \rightarrow \infty} \frac{L_{k}(z)}{z}=\frac{1-\varepsilon}{1-\rho(k)}
$$


The proof of the lower bound is completed by letting $\varepsilon \rightarrow 0$ and $k \rightarrow \infty$.

We now move to the upper bound. For all $P \in$ SMART and $P=F B$,

$$
V(x)^{\mathrm{P}} \leq_{s t} L(x+Q),
$$

where $Q$ is the steady state work in the system upon customer arrivals. Again using a PLCFS system, we observe that the events $\left\{S_{y}(t)-Q>x\right\}$ and $\left\{L_{y}(x+Q)<t\right\}$ coincide. Arguing as above, and using that

$$
\lim _{t \rightarrow \infty} \frac{S_{y}(t)-Q}{t}=1-\rho(y)
$$

completes the proof of the upper bound.

It is not surprising that Condition 1 holds for $\mathrm{P}=\mathrm{FB}$ and $\mathrm{P} \in \mathrm{SMART}$ : as noted in Núnez Queija (2002), it seems that such a result holds for all policies under which the system remains stable when a permanent customer is added, including FB and all SMART policies. The intuition is that over the time interval $[0, \infty)$, the long-run fraction of service capacity devoted to non-permanent customers must converge to $\rho$ for the system to remain stable; thus a permanent customer must receive a fraction $1-\rho$ of the service capacity while it is in the system.

Before proving that Condition 2 holds for SMART and FB, we prove an auxiliary result. A similar result has been shown before for the workload in the $M / G / 1$ queue in Jelenkovic and Momcilovic (2003). A key ingredient to the proof of this auxiliary result is the following lemma, which is due to Resnick and Samorodnitsky (1999). We use the notation $x^{+}=\max \{x, 0\}$.

Lemma 15 Let $S_{n}=X_{1}+\cdots+X_{n}$ be a random walk with i.i.d. step sizes such that $E\left[X_{1}\right]<0$ and $E\left[\left(X_{1}^{+}\right)^{p}\right]<\infty$ for some $p>1$. Then, for any $\alpha<\infty$, there exist $c, k^{*}>0$ such that for any $n, x$ and $k>k^{*}$,

$$
P\left(S_{n}>k x \mid X_{i}<x, i \leq n\right) \leq c x^{-\alpha} .
$$

Lemma 16 Let $X_{1}, X_{2}, \ldots$ be i.i.d. random variables with $E\left[\left(X_{1}^{+}\right)^{p}\right]<\infty$ for some $p>1$. Let $S_{n}(y)=\sum_{i=1}^{n}\left(X_{i} \wedge y\right)$. Define $M(y)=\sup _{n} S_{n}(y)$. For every $\beta>0$, there exists a $k>0$ such that $P(M(x)>k x)=\mathrm{o}\left(x^{-\beta}\right)$.

Proof Let $\beta>0$. For fixed $y \geq 1$, we write the standard geometric random sum decomposition

$$
M(y) \stackrel{d}{=} \sum_{i=1}^{N(y)} H_{i}(y)
$$


with $N(y)$ the number of ladder heights, and $H_{i}(y)$ the $i$ th overshoot; for details see e.g. Chapter VIII of Asmussen (2003). The variable $y$ simply indicates that the underlying random variables $X_{i}$ are truncated at $y$.

By a sample-path comparison, it follows that

$$
M(y) \stackrel{s t}{\leq} \sum_{i=1}^{N(\infty)}\left[H_{i}(\infty) \wedge y\right]
$$

Writing $H_{i}=H_{i}(\infty)$, we have for any $k, \gamma>0$,

$$
P(M(x)>k x) \leq P\left(N(\infty)>\left\lfloor x^{\gamma}\right\rfloor\right)+P\left(\sum_{i=1}^{\left\lfloor x^{\gamma}\right\rfloor}\left(H_{i} \wedge x\right)>k x\right) .
$$

Since the number of overshoots is geometrically distributed, the first term in (15) behaves like $\exp \left(-c\left\lfloor x^{\gamma}\right\rfloor\right)$ for some $c>0$. Since this decays faster than any power tail for any $\gamma>0$, it suffices to consider the second term.

Let $0<q<\min \{1, p-1\}$. Since the tail of $H_{i}$ is one degree heavier than that of the $X_{k}$ (see Theorem 2.1 in Chapter VIII of Asmussen (2003)), we have $E H_{i}^{q}<E H_{i}^{p-1}<\infty$. Hence, $H_{i}^{q}-2 E\left[H_{i}^{q}\right]$ satisfies the assumption of Lemma 15. Take $\gamma \in(0, q)$. Since $y^{q}$ is a concave function in $y$, we have

$$
\begin{aligned}
P\left(\sum_{i=1}^{\left\lfloor x^{\gamma}\right\rfloor}\left(H_{i} \wedge x\right)>k x\right) & =P\left(\left[\sum_{i=1}^{\left\lfloor x^{\gamma}\right\rfloor}\left(H_{i} \wedge x\right)\right]^{q}>(k x)^{q}\right) \\
& \leq P\left(\sum_{i=1}^{\left\lfloor x^{\gamma}\right\rfloor}\left(H_{i} \wedge x\right)^{q}>(k x)^{q}\right) \\
& =P\left(\sum_{i=1}^{\left\lfloor x^{\gamma}\right\rfloor}\left(\left(H_{i} \wedge x\right)^{q}-2 E\left[H_{i}^{q}\right]\right)>(k x)^{q}-2\left\lfloor x^{\gamma}\right\rfloor E\left[H_{i}^{q}\right]\right) .
\end{aligned}
$$

In order to apply Lemma 15, we rewrite (16) to replace the truncated random variables $\left(H_{i} \wedge x\right)$ by random variables that are conditioned to be smaller than a certain value. Choose an integer $l>\beta /(q-\gamma)$. Considering the event that at least $l$ of the $H_{i}$ are larger than $x$, and its complement, we find

$$
\begin{aligned}
& P\left(\sum_{i=1}^{\left\lfloor x^{\gamma}\right\rfloor}\left(\left(H_{i} \wedge x\right)^{q}-2 E\left[H_{i}^{q}\right]\right)>(k x)^{q}-2\left\lfloor x^{\gamma}\right\rfloor E\left[H_{i}^{q}\right]\right) \\
& \quad \leq\left(\begin{array}{c}
\left\lfloor x^{\gamma}\right\rfloor \\
l
\end{array}\right) P\left(H_{i}>x\right)^{l}+P\left(\sum_{i=1}^{\left\lfloor x^{\gamma}\right\rfloor}\left(H_{i}^{q}-2 E\left[H_{i}^{q}\right]\right)>(k x)^{q}-2\left\lfloor x^{\gamma}\right\rfloor E\left[H_{i}^{q}\right] \mid \#\left\{i: H_{i}>x\right\}<l\right) \\
& \quad \leq x^{\gamma l} P\left(H_{i}>x\right)^{l}+P\left(\sum_{i=1}^{\left\lfloor x^{\gamma}\right\rfloor-l}\left(H_{i}^{q}-2 E\left[H_{i}^{q}\right]\right)>(k x)^{q}-l x^{q}-2\left\lfloor x^{\gamma}\right\rfloor E\left[H_{i}^{q}\right] \mid H_{i} \leq x\right)
\end{aligned}
$$


Here $\# V$ denotes the number of elements in the set $V$. We complete the proof by showing that both terms in (17) are $o\left(x^{-\beta}\right)$. Since $E\left[H_{i}^{q}\right]<\infty$, we know that $P\left(H_{i}>x\right)=o\left(x^{-q}\right)$. Hence, since $0<\gamma<q$, and $l>\beta /(q-\gamma)$, we have $x^{\gamma l} P\left(H_{i}>x\right)^{l}=o\left(x^{\gamma l} x^{-q l}\right)=o\left(x^{-\beta}\right)$. Let $\bar{k}>0$. Since $q>\gamma$, for $k$ large enough, the second term in (17) is smaller than

$$
P\left(\sum_{i=1}^{\left\lfloor x^{\gamma}\right\rfloor-l}\left(H_{i}^{q}-2 E\left[H_{i}^{q}\right]\right)>\bar{k}\left(x^{q}-2 E\left[H_{i}^{q}\right]\right) \mid H_{i}^{q}-2 E\left[H_{i}^{q}\right] \leq x^{q}-2 E\left[H_{i}^{q}\right]\right) .
$$

Applying Lemma 15 with a suitable choice of $\bar{k}$, there exist $c>0$ and $\eta>\beta / q$ such that (18) is smaller than

$$
c\left(x^{q}-2 E\left[H_{i}^{q}\right]\right)^{-\eta} \sim c\left(x^{q}\right)^{-\eta}=o\left(x^{-\beta}\right), \quad x \rightarrow \infty .
$$

This completes the proof.

Consider now a $G I / G I / 1$ queue with the same interarrival-time distribution as before, but with generic service time $B \wedge x$. Set $A_{x}(t)=\sum_{i=1}^{K(t)}\left(B_{i} \wedge x\right)$, with $K(t)$ the number of arrivals in $(0, t]$, so $A_{x}(t)$ is the work entering the queue in the time interval $(0, t]$. Furthermore, at the beginning of each busy period an initial setup time $x$ is added. Let $\widetilde{L}_{x}^{* s}$ be the residual busy period after the arrival of a customer of size $x$. Then $\widetilde{L}_{x}^{* s}$ can be represented as follows:

$$
\widetilde{L}_{x}^{* s}=\inf \left\{t: x+\widetilde{Q}_{x}^{s}+A_{x}(t)-t=0\right\},
$$

with $\widetilde{Q}_{x}^{s}$ the steady-state workload upon customer arrivals in this queue, including the effect of the initial set-up.

Furthermore, let $D_{x}^{\mathrm{P}}(t)$ be the stochastic processes of work under policy $\mathrm{P}$ that would have priority over an arriving job of size $x$ at time $t$. In other words: $D_{x}^{\mathrm{P}}(t)$ is distributed like $V_{t}(x)^{\mathrm{P}}-x$ given that there are no arrivals after time $t$, where $V_{t}(x)^{\mathrm{P}}$ is the sojourn time for a job of size $x$ arriving at time $t$ under policy $\mathrm{P}$.

Lemma 17 For $\mathrm{P}=\mathrm{FB}$ and all $\mathrm{P} \in \mathrm{SMART}$, we have

$$
V(x)^{\mathrm{P}} \leq_{s t} \widetilde{L}_{x}^{* s}
$$

Proof The bound holds for FB, since the residual busy period bounds $V(x)^{\mathrm{FB}}$ if the setup time were not included.

To see that the residual busy period also bounds $V(x)^{\mathrm{P}}$ for $\mathrm{P} \in$ SMART, note that the process $D_{x}^{\mathrm{P}}(t)$ consists of two types of busy periods: (i) busy periods started by a job of original size $>x$ that now has remaining size $\leq x$ and (ii) busy periods started by a job of original size $\leq x$. Note that the Consistency and Transitivity Properties prevent two jobs in the system with original size 
larger than $x$ from obtaining a remaining size smaller than $x$ at the same instant. In both cases, the Bias Property prevents any job with remaining size $>x$ from receiving service during the busy period; thus only new arrivals of size $\leq x$ can contribute once the busy period is started. Since the initial job under P $\in$ SMART is necessarily smaller than the setup $x$ of $\widetilde{L}_{x}^{* s}$, and the arrivals during the busy period are stochastically larger in $\widetilde{L}_{x}^{* s}$, the residual length of both of these busy periods is stochastically smaller than $\widetilde{L}_{x}^{* s}$.

The following lemma implies that Condition 2 holds for FB and SMART .

Lemma 18 For every $\beta>0$, there exists a constant $k$ such that

$$
P\left(V(x)^{\mathrm{P}}>k x\right)=o\left(x^{-\beta}\right), \quad x \rightarrow \infty
$$

for all $\mathrm{P} \in \mathrm{SMART}$ and for $\mathrm{P}=\mathrm{FB}$. As a consequence, Condition 2 holds for $\mathrm{P}=\mathrm{FB}$ and $\mathrm{P} \in \mathrm{SMART}$.

Proof Let $\mathrm{P}=\mathrm{FB}$ or $\mathrm{P} \in \mathrm{SMART}$. We will bound $V(x)^{\mathrm{P}}$ using the residual busy period $\widetilde{L}_{x}^{* s}$ as per Lemma 17. Furthermore, define

$$
U_{x}^{c} \stackrel{\text { def }}{=} \sup _{t>0}\left[A_{x}(t)-c t+x\right]=x+\sup _{t>0}\left[A_{x}(t)-c t\right]
$$

Then $U_{x}^{1}=\widetilde{Q}_{x}^{s}$. For $(1-\rho) / 2<\delta<1 / 2$ and $k>1 / \delta$, we have by Lemma 17 ,

$$
\begin{aligned}
P\left(V(x)^{\mathrm{P}}>k x\right) & \leq P\left(\widetilde{L}_{x}^{* s}>k x\right) \\
& \leq P\left(x+U_{x}^{1}+A_{x}(k x)-k x>0\right) \\
& \leq P\left(U_{x}^{1}+A_{x}(k x)-(1-2 \delta) k x+x>\delta k x\right) \\
& \leq P\left(U_{x}^{1}>\delta k x / 2\right)+P\left(A_{x}(k x)-(1-2 \delta) k x+x>\delta k x / 2\right) \\
& \leq P\left(U_{x}^{1-2 \delta}>\delta k x / 2\right)+P\left(\sup _{t>0}\left[A_{x}(t)-(1-2 \delta) t+x\right]>\delta k x / 2\right) \\
& =2 P\left(U_{x}^{1-2 \delta}>\delta k x / 2\right) .
\end{aligned}
$$

By taking $\bar{k}=k \delta / 2$, and $c=1-2 \delta$, it suffices to show that there exists a $\bar{k}>1$ such that

$$
P\left(U_{x}^{c}>\bar{k} x\right)=P\left(U_{x}^{c}-x>(\bar{k}-1) x\right)=o\left(x^{-\beta}\right) .
$$

We complete the proof by viewing $U_{x}^{c}-x$ in terms of a random walk. Since the supremum in (20) is attained at arrival instants, we may write

$$
U_{x}^{c}-x=\sup _{n} \sum_{i=1}^{n}\left(B_{i} \wedge x\right)-c A_{i} \leq \sup _{n} \sum_{i=1}^{n}\left[\left(B_{i}-c A_{i}\right) \wedge x\right]
$$


where $A_{i}$ is the time between the $(i-1)$ st arrival and the $i$ th arrival. Since $E\left[B_{i}-(1-2 \delta) A_{i}\right]<$ $E\left[B_{i}-\rho A_{i}\right]=0$ and $E\left[\left(\left(B_{i}-(1-2 \delta) A_{i}\right)^{+}\right)^{p}\right] \leq E\left[B_{i}^{p}\right]<\infty$, we may apply Lemma 16 , and (21) follows.

To show that $\mathrm{FB}$ and $\mathrm{P} \in \mathrm{SMART}$ obey Condition 2 , note that since $\bar{F} \in \mathcal{I} \mathcal{R}$, there exists a $\beta>0$ such that $x^{-\beta}=o(P(B>x))$. Take this $\beta$ and choose $k$ as in (19). Condition 2 now follows.

Proof of Theorem 3 Combining Lemmas 14 and 18 guarantees that FB and all P $\in$ SMART obey Conditions 1 and 2. The theorem then follows from applying Theorem 13.

\section{Stochastic bounds for $\mathrm{M} / \mathrm{GI} / 1$}

In this section we derive the stochastic bounds on the sojourn time of SMART policies given in Theorem 6. The following properties are necessary in the proofs: PASTA (Poisson Arrivals See Time Averages) and the linearity of busy periods, i.e., $L(x+y) \stackrel{d}{=} L(x)+L(y)$. Since these properties depend on memoryless arrivals, we limit ourselves to the M/GI/1 setting.

We first consider the quantities $D_{x}^{\mathrm{FB}}(t)$ and $D_{x}^{\mathrm{SRPT}}(t)$, which play a crucial role in this section. Remember that $D_{x}^{\mathrm{P}}(t)$ is the stochastic process of work under policy P that would have priority over an arriving job of size $x$ at time $t$. Denote by $D_{x}^{\mathrm{P}}$ its stationary version. Note that under SMART policies, $D_{x}^{\mathrm{P}}$ may in general depend on the behavior of the system after $x$ arrives. However, it was shown in Wierman, Harchol-Balter and Osogami (2005) that $D_{x}^{\mathrm{P}} \leq_{s t} D_{x}^{\mathrm{SRPT}}$, and $D_{x}^{\mathrm{SRPT}}$ depends only on the state of the system at the arrival of the tagged job.

Let us now describe $D_{x}^{\mathrm{FB}}(t)$ and $D_{x}^{\mathrm{SRPT}}(t)$. The process $D_{x}^{\mathrm{FB}}(t)$ is simple: it is the work(load) process of an M/GI/1 queue with service times distributed like $B \wedge x$ and arrival rate $\lambda$. The process $D_{x}^{\mathrm{SRPT}}(t)$ is more complex. Under SRPT, only arrivals of size smaller than or equal to $x$ (call these small jobs) immediately add their size to $D_{x}^{\mathrm{SRPT}}(t)$. An arrival of size larger than $x$ (call this a large job) will contribute $x$ to $D_{x}^{\mathrm{SRPT}}(t)$ the moment its remaining size drops to $x$. Thus, small arrivals form a Poisson process with rate $\lambda$ and service distribution $B I(B \leq x)$, but large arrivals do not form a Poisson process. In fact, a large arrival can only add to $D_{x}^{\mathrm{SRPT}}(t)$ when $D_{x}^{\mathrm{SRPT}}(t)=0$. While $D_{x}^{\mathrm{SRPT}}(t)>0$, the only arrivals are small arrivals, so the arrival process during those periods is Poisson.

The processes $D_{x}^{\mathrm{SRPT}}(t)$ and $D_{x}^{\mathrm{FB}}(t)$ are both work-conserving, so we can view them as the work processes of PLCFS systems with the same job sizes, but (possibly) different arrival times. We refer to these PLCFS systems as the transformed FB and SRPT systems in order to emphasize that the scheduling is PLCFS (not SRPT and FB). Note that every arrival to the original system will eventually contribute $B \wedge x$ work to both transformed systems. 


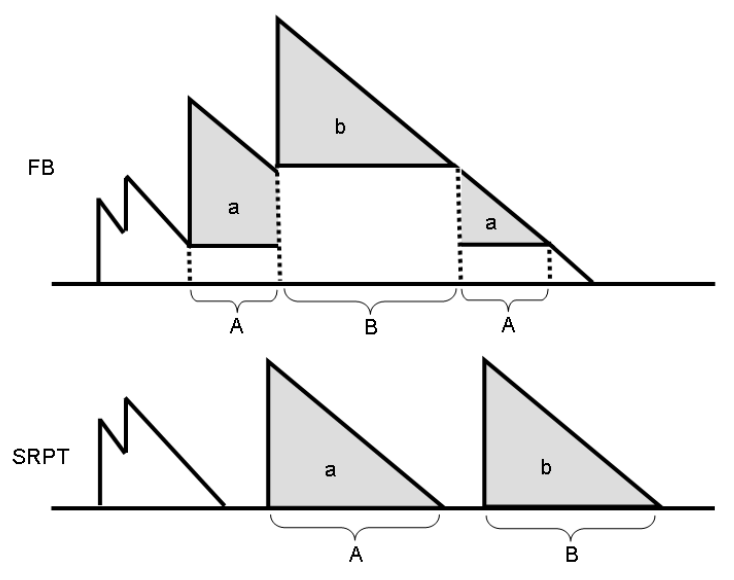

Figure 3: This diagram illustrates the main idea in the proof of Proposition 20. The two figures show $D_{x}^{\mathrm{P}}(t)$ under $\mathrm{FB}$ and SRPT. The shaded jobs are large jobs. We have argued that the arrival processes are stochastically identical while large jobs are in the system, so none of these arrivals are drawn. In the proof, we pair up the times when large job a is the most recent large arrival and the times when job $b$ is the most recent large arrival. This figure illustrates that, since all large jobs arrive when $D_{x}^{\mathrm{P}}=0$ under $\mathrm{SRPT}, D_{x}^{\mathrm{SRPT}} \leq_{s t} D_{x}^{\mathrm{FB}}$ given that a large job is in the system.

Let us make a few observations about the sojourn times of large jobs in the two transformed systems. In the transformed SRPT system, the sojourn time of a large job is stochastically equal to $L_{x}(x)$, while in the transformed FB system, it is distributed like $\widetilde{L_{x}}(x)$. However, the following lemma states that the time that a large job is the most recent large arrival in the transformed FB system is distributed like $L_{x}(x)$, just as for the transformed SRPT system.

Lemma 19 Let $M$ denote the time that a tagged large job is the most recent large arrival present in the $M / G / 1 /$ PLCFS queue. Then $M \stackrel{d}{=} L_{x}(x)$.

Proof During the sojourn time of the tagged job of size $x$, the arrival of another job of size $x$ just creates a sub-busy period that does not contribute. Since the arrival process is Poisson and all small arrivals contribute to $M$, we have $M \stackrel{d}{=} L_{x}(x)$.

This allows us to pair up the times of the most recent large arrivals in the system. This pairing is illustrated in Figure 3. Using this pairing, we prove the following proposition.

Proposition 20 In the $M / G / 1$ queue, we have $D_{x}^{\mathrm{P}} \leq_{s t} D_{x}^{\mathrm{SRPT}} \leq_{s t} D_{x}^{\mathrm{FB}}$ for all $x$ and all $\mathrm{P} \in \mathrm{SMART}$.

Proof The first inequality was proven in Theorem 4.1 in Wierman, Harchol-Balter and Osogami (2005). 
To prove the second inequality, we first compare $D_{x}^{\mathrm{FB}}$ and $D_{x}^{\mathrm{SRPT}}$ conditional on the presence of a large job, using the pairing described above. In the transformed SRPT system, $D_{x}^{\operatorname{SRPT}}(t)=0$ at every time $t$ when a large job arrives, whereas large jobs arrive to the transformed FB system at Poisson points, at which the transformed system is not necessarily idle. We can conclude that conditional on the presence of a large arrival in the transformed system, $D_{x}^{\mathrm{SRPT}} \leq_{s t} D_{x}^{\mathrm{FB}}$.

Second, when no large arrival is in the system, the transformed FB and SRPT systems have stochastically identical work processes: only small jobs arrive, according to a Poisson process. Since the periods that large jobs are in the system are stochastically identical in length, the same holds for the periods that no large jobs are in system. Since both systems will achieve steady state, it must hold that $D_{x}^{\mathrm{SRPT}} \leq_{s t} D_{x}^{\mathrm{FB}}$. Since we are only concerned with the work seen by a Poisson arrival $x$ to a regenerative system that is convergent, we can apply PASTA, see Wolff (1989). This completes the proof.

To complete the proof of Theorem 6, we shall use the following characterization of the conditional sojourn time under SRPT, PSJF, and FB. Under SRPT, the waiting time of a job of size $x$ is distributed like a busy period with an initial customer of size $D_{x}^{\mathrm{SRPT}}$ and generic service time $B I(B<x)$, i.e.,

$$
W(x)^{\mathrm{SRPT}} \stackrel{d}{=} L_{x}\left(D_{x}^{\mathrm{SRPT}}\right) .
$$

Under PSJF, the service of a job is interrupted only by all jobs with original size smaller than $x$, the residence time is distributed like a busy period with an initial job of size $x$ :

$$
R(x)^{\mathrm{PSJF}} \stackrel{d}{=} L_{x}(x) .
$$

Under FB, the sojourn time of a job of size $x$ is stochastically equal to the length of a busy period with generic service time $B \wedge x$ and an initial customer of size $x+D_{x}^{\mathrm{FB}}$ :

$$
V(x)^{\mathrm{FB}} \stackrel{d}{=} \widetilde{L}_{x}\left(x+D_{x}^{\mathrm{FB}}\right) .
$$

We are now ready to give the proof of Theorem 6 .

Proof of Theorem 6 Theorem 4.1 in Wierman, Harchol-Balter and Osogami (2005) states that

$$
V(x)^{\mathrm{P}} \leq_{s t} L_{x}\left(x+D_{x}^{\mathrm{SRPT}}\right) .
$$

Since $L_{x}(Y) \leq s t \widetilde{L_{x}}(Y)$ for all random variables $Y$, Proposition 20 implies

$$
V(x)^{\mathrm{P}} \leq_{s t} L_{x}\left(x+D_{x}^{\mathrm{SRPT}}\right) \leq_{s t} \widetilde{L_{x}}\left(x+D_{x}^{\mathrm{FB}}\right) .
$$

The proof of the theorem is completed by rewriting (25) using the linearity of busy periods and (22), (23), and (24). 


\section{Conclusion}

The SMART classification represents an emerging style of research based on analyzing large groups of policies instead of individual disciplines. Recent papers such as Núñez Queija (2002), Wierman and Harchol-Balter (2003, 2005), and Wierman, Harchol-Balter and Osogami (2005), have attempted to uncover the effect of general scheduling heuristics and mechanisms on performance, thus adding structure to the space of scheduling policies that cannot be obtained through the analysis of individual policies. Beyond the theoretical motivation for studying classifications of scheduling policies, there are practical reasons. Namely, in practice, system designers can never implement the idealized policies (such as SRPT, PS, and FB) that are the focus of theoretical research. By analyzing classifications of policies, the hope is that theoretical results can be obtained for the unique, hybrid policies that are actually implemented in practice.

In this paper, we have analyzed the GI/GI/1 tail behavior of the sojourn time under both FB and SMART policies. We have proven that both FB and SMART policies have (near) optimal sojourn-time tails under heavy-tailed service distributions, and still outperform FCFS under light-tailed service distributions provided the service time of a customer is not too large. These analyses can be viewed as a formal verification that the heuristic of "biasing toward small jobs" is appropriate for many computer system applications, where service distributions tend to be heavy-tailed. Furthermore, we have derived stochastic bounds that relate the conditional sojourn times of FB and SMART policies in the $\mathrm{M} / \mathrm{GI} / 1$ setting.

Acknowledgements We would like to thank the referees for their suggestions and comments, which have improved the presentation and readability of the paper.

\section{References}

S. Aalto, U. Ayesta, and E. Nyberg-Oksanen. 2004. Two-level processor-sharing scheduling disciplines: Mean delay analysis. In Proceedings of ACM Sigmetrics-Performance.

S. Asmussen. 2003. Applied Probability and Queues, second edition. Springer, New York.

J. Abate and W. Whitt. 1997. Asymptotics for $M / G / 1$ low-priority waiting-time tail probabilities. Queueing Systems 25, 173-233.

P. Barford and M. Crovella. 1998. Generating representative web workloads for network and server performance evaluation. In Proceedings of ACM Sigmetrics. 
S. Borst, O. Boxma, R. Núñez Queija, and B. Zwart. 2003. The impact of the service discipline on delay asymptotics. Performance Evaluation 54, 175-206.

S. Borst, R. Núñez Queija, and B. Zwart. 2006. Sojourn time asymptotics in Processor Sharing queues. Queueing Systems 53, 31-51.

L. Cherkasova. 1998. Scheduling strategies to improve response time for web applications. In High-performance computing and networking: international conference and exhibition, 305314.

A. Downey. 2001. Evidence for long-tailed distributions in the internet. In Proceedings of ACM SIGCOMM Internet Measurement Workshop.

F. Guillemin, Ph. Robert, and B. Zwart. 2004. Tail asymptotics for processor sharing queues. Advances in Applied Probability 36, 525-543.

M. Harchol-Balter, B. Schroeder, N. Bansal, and M. Agrawal. 2003. Implementation of SRPT scheduling in web servers. ACM Transactions on Computer Systems, 21(2).

P. Jelenkovic and P. Momcilovic. 2003. Large deviation analysis of subexponential waiting times in a Processor Sharing queue. Mathematics of Operations Research 28, 587-608.

W. Leland, M. Taqqu, W. Willinger, and D. Wilson. 1993. On the self-similar nature of ethernet traffic. In Proceedings of SIGCOMM '93, 183-193.

M. Mandjes and M. Nuyens. 2005. Sojourn times in the M/G/1 FB queue with light-tailed service times. Probability in the engineering and informational sciences 19, 351-361.

M. Mandjes and B. Zwart. 2006. Large deviations for waiting times in processor sharing queues. Queueing Systems 52, 237-250.

D. McWherter, B. Schroeder, N. Ailamaki, and M. Harchol-Balter. 2004. Priority mechanisms for OLTP and transactional web applications. In International Conference on Data Engineering.

R. Núñez Queija. 2002. Queues with equally heavy sojourn time and service requirement distributions. Annals of Operations Research 113, 101-117.

M. Nuyens and B. Zwart. (2005). A large-deviations analysis of the GI/GI/1 SRPT queue. Queueing Systems, to appear.

Preprint version available at http://www.few.vu.nl/ mnuyens/publications/srpt.html

M. Nuyens. 2004. The Foreground-Background Queue. PhD thesis, University of Amsterdam. 
D. Peterson. 1996. Data center I/O patterns and power laws. In CMG Proceedings.

I. Rai, G. Urvoy-Keller, M. Vernon, and E. Biersack. 2004. Performance modeling of LAS based scheduling in packet switched networks. In Proceedings of ACM Sigmetrics-Performance.

M. Rawat and A. Kshemkalyani. 2003. SWIFT: Scheduling in web servers for fast response time. In Symposium on Network Computing and Applications.

S. Resnick and G. Samorodnitsky. 1999. Activity periods of an infinite server queue and performance of certain heavy tailed fluid queues. Queueing Systems 33, 43-71.

R. Righter and J. Shanthikumar. 1989. Scheduling multiclass single server queueing systems to stochastically maximize the number of successful departures Probability in the Engineering and the Informational Sciences 3, 323-333.

R. Righter and J. Shanthikumar. 1992. Extremal properties of the FIFO discipline in queueing networks. Journal of Applied Probability 29, 967-978.

A. Wierman and M. Harchol-Balter. 2003. Classifying scheduling policies with respect to unfairness in an M/GI/1. In Proceedings of ACM Sigmetrics.

A. Wierman and M. Harchol-Balter. 2005 Classifying scheduling policies with respect to higher moments of conditional response time. In Proceedings of ACM Sigmetrics.

A. Wierman, M. Harchol-Balter, and T. Osogami. 2005. Nearly insensitive bounds for SMART scheduling. In Proceedings of ACM Sigmetrics.

R. Wolff. 1989 Stochastic Modeling and the Theory of Queues. Prentice Hall.

S. Yang and G. de Veciana. 2004. Enhancing both network and user performance for networks supporting best effort traffic. Transactions on Networking 12, 349-360. 